\title{
Early time evolution of Freèdericks patterns generated from states of electroconvection
}

\author{
Denis Funfschilling* and Michael Dennin \\ Department of Physics and Astronomy, University of California at Irvine, Irvine, California 92697-4575 and \\ *current address: Department of Physics and Astronomy, \\ University of California at Santa Barbara, Santa Barbara, California
}

(Dated: November 20, 2018)

\begin{abstract}
We report on the early time ordering in a nematic liquid crystal subjected to a sudden change in external ac electric field. We compare time evolution for two different initial states of electroconvection. Electroconvection is a highly driven state of a nematic liquid crystal involving convective motion of the fluid and periodic variations of the molecular alignment. By suddenly changing either the voltage or the frequency of the applied ac field, the system is brought to the same thermodynamic conditions. The time ordering of the system is characterized by the evolution of features of the power spectrum, including the average wavenumber, total power, and shape of the the power spectrum. The differences between the two classes of quenches are discussed, as well as the possibility of scaling behavior during this initial phase of domain growth.

PACS numbers: 89.75.Da,47.54.+r,64.70.Md
\end{abstract}

Understanding systems driven far from equilibrium remains one of the outstanding challenges of contemporary physics. At the heart of the issue is the lack of any single principle that is equivalent to the minimization of free energy that is applicable in thermodynamic equilibrium [1]. A subset of this larger issue is the question of the transition between states of a system after a sudden change in an external parameter, or a quench. The general question of the behavior of systems after a quench is often referred to as phase ordering or coarsening 2], as domains of the new steady state of the system order and grow.

Classically, phase ordering has been studied in the context of the transition between two equilibrium states. In this case, minimization of a free energy plays a central role in understanding the ordering process [2]. More recently, transitions between driven states of a system have gained interest [3, 4, 5, 6, 7, 8, 9, 10, 11, 12. In this case, one considers the ordering of steady-states of a driven system after the driving force has been changed. The free energy does not play a role. However, there is growing evidence that the dynamics of topological defects govern the phase ordering in both equilibrium and nonequilibrium systems 2, 12, 13]. One system that is useful for studying quenches in driven systems is electroconvection 10, 11, 12.

Electroconvection occurs when a nematic liquid crystal is placed between two plates and an ac electric voltage is applied perpendicular to the plates [14, 15]. A nematic liquid crystal consists of long, rod-like molecules that on average are aligned along a fixed axis, which is referred to as the director. In the absence of electroconvection, the director is spatially uniform. Above a critical voltage, the director develops a periodic spatial variation, and there is an associated periodic flow, or convection rolls. The most common geometry for electroconvection is to have the director parallel to the glass plates initially. An interesting case exists when the director is initially perpendicular to the plates, also known as homeotropic alignment [16, 17].

An important parameter that is used to characterize nematic liquid crystals is the dielectric anisotropy [15]. For an anisotropic material, such as a nematic liquid crystal, the dielectric constant is a tensor. The dielectric anisotropy is the difference between the dielectric constant for the case where the electric field and director are perpendicular and the case where they are parallel. For materials with a negative dielectric anisotropy in a homeotropic configuration, the initial transition as a function of applied voltage is the Freèdericks transition, which is followed by the transition to electroconvection 16, 17]. The Freèdericks transition corresponds to the director developing a tilt relative to its initial alignment perpendicular to the plates. This state is an equilibrium state in which the angle of tilt is fixed for a given value of the voltage. However, there is a degeneracy due to the fact that the director tilt can adopt any azimuthal angle. Regions with the same azimuthal angle are usually referred to as Freèdericks domains. Once the Freèdericks transition has occurred, there is a component of the director parallel to the plates. Therefore, there is a second critical voltage at which electroconvection will occur.

The Freèdericks transition is independent of frequency; whereas, the critical voltage for electroconvection is frequency dependent. This allows for exploration of two different types of quenches to the same equilibrium state. With different starting points, either a rapid change in frequency or voltage can bring the system to the same final frequency and voltage, and hence the same Freèdericks state. However, the system evolution to reach this final state is different. For a quench down in voltage, the average director tilt angle has to relax to the correct value. For a quench in frequency, the average tilt angle of director should already have the appropriate value. In both cases, the pattern present in the director field, charge distribution, and flow field, due to electroconvection have to relax. 
In this paper, we report on a comparison of these two quenches. We focus on the initial dynamics from the time of the quench until the point where the spatial patterns are similar. The system's evolution is characterized by a number of measures based on the power spectrum of the images. It should be noted that it is also interesting to consider the late-time evolution of the system. As the Freèdericks state is an equilibrium state, the latetime evolution should be the same for both systems [2]. However, large-scale imperfections in the system complicate studies of the late-time dynamics, for which large aspects ratios are critical. Therefore, the question of the late-time behavior will be the subject of future work.

For these experiments, homeotropic cells of the nematic liquid crystal N4 were used. The liquid crystal N4 is a eutectic mixture of the two isomers of 4-methoxy-4'-n-butylazoxybenzene $\left(\mathrm{CH}_{3} \mathrm{O}-\mathrm{C}_{6} \mathrm{H}_{4}-\mathrm{NON}-\mathrm{C}_{6} \mathrm{H}_{4}-\mathrm{C}_{4} \mathrm{H}_{9} \quad\right.$ and $\left.\mathrm{CH}_{3} \mathrm{OC}_{6} \mathrm{H}_{4}-\mathrm{NNO}-\mathrm{C}_{6} \mathrm{H}_{4}-\mathrm{C}_{4} \mathrm{H}_{9}\right)$. It was obtained from EM Industries (a Merck company), now EMD Chemicals Inc. 18]. The N4 was used without further purification. The method for obtaining homeotropic alignment is described in detail in Ref. [19]. Briefly, a surfactant coating is made on ITO coated glass using a Langmuir-Blodgett technique. A mixture of $43 \%$ of $\mathrm{N} 4$ and $57 \%$ arachidic acid (C20) diluted in chloroform is spread at the air-water interface, forming a monolayer that was compressed to $10 \mathrm{mN} / \mathrm{m}$. We used C20 obtained from Sigma-Aldrich with a quoted purity of $\geq 99 \%$. It was used without further purification. The pressure was held constant while ITO glass is coated with 10 layers of the Langmuir monolayer. After the coating of the surface, the glass is baked in an oven at a temperature of $50{ }^{\circ} \mathrm{C}$. A $25 \mu \mathrm{m}$ mylar spacer is placed between the two ITO glasses. Two opposite sides of the cell are sealed with epoxy. The cell is filled with N4 by capillary action. The two remaining sides of the cell are sealed with 5 minute epoxy.

The details of the experimental apparatus are described in Ref. 20]. Essentially, there is a temperature control stage that holds the sample at a constant temperature within $\pm 5 \mathrm{mK}$. The sample is illuminated from below and imaged from above using standard shadowgraph techniques. A pair of crossed-polarizers (one below the sample and one above the sample) allow for simultaneous imaging of electroconvection patterns and of Freédericksz domains. The use of ITO coated glass allows for the application of an ac voltage to the sample.

The general state diagram for homeotropic samples of N4 is reported in Ref. [19]. There is the expected Freèdericks transition at approximately $V_{c F}=9.3 \mathrm{~V}$, independent of applied frequency. The transition to electroconvection is frequency dependent. The solid black horizontal and vertical lines with triangular endpoints in Fig. 1 illustrate the two types of quenches that are reported on in this paper. The solid curve is the transition to electroconvection, and the dashed black curve is the critical voltage for the Freèdericks transition (both curves

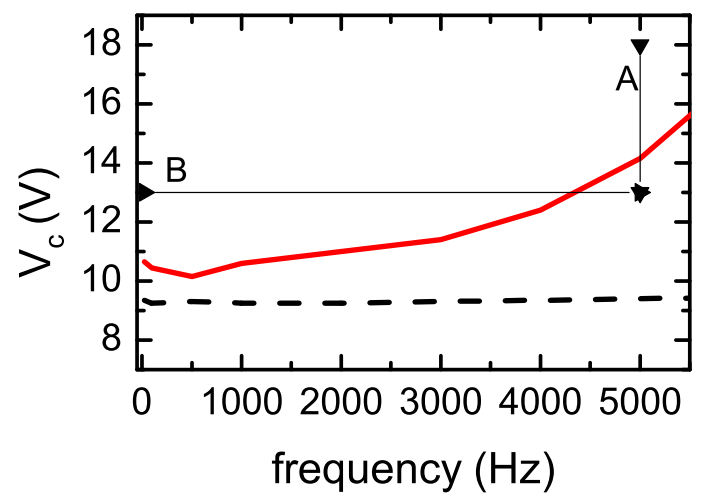

FIG. 1: A portion of the phase diagram for homeotropic N4 as reported in Ref. 19]. The dashed curve is the critical voltage for the Freédericksz transition. The solid curve is the critical voltage for the transition to electroconvection. The horizontal and vertical lines connect the starting and ending point of the two quenches.

were reported in Ref. [19]). Both quenches are selected to have the same final equilibrium conditions of applied voltage and frequency within the regime where Freèdericks domains exist, but electroconvection does not. The two starting points represent different initial states for the system. The quench labelled A starts from a chaotic electroconvection state and uses a change in voltage. Physically, this means the tilt of the director must change as part of the quench. The path labelled B starts from a regular state of electroconvection and involves a change in frequency. In this case, the distance from the Freèdericks critical voltage is not changed. Therefore, the details of the initial evolution should be different.

For discussing these quenches, it is important to define a few parameters. We will use $\epsilon=\left(V / V_{c}\right)^{2}-1$ to refer to the distance from the electroconvection critical voltage $\left(V_{c}\right)$ at a fixed frequency. Therefore, since all of the quenches end at a frequency $f=5000 \mathrm{~Hz}$ and a rms voltage of $V=13.0 \mathrm{~V}$, the final point for the quenches is $\epsilon=-0.156$ and $f=5000 \mathrm{~Hz}$. The two starting points are $\epsilon=0.618$ with $f=5000 \mathrm{~Hz}$ and $\epsilon=0.490$ with $f=25 \mathrm{~Hz}$.

To provide a framework for the two quenches, we have characterized the final state both by making quenches from below the Freèdericks critical voltage and by slowing stepping to the final state. These studies involving increasing the voltage established the existence of largescale spatial inhomogeneities in the Freèdericks domains. Essentially the same spatial pattern is obtained either by small steps in voltage or by considering the late-time state after a large change in voltage. The spatial inhomogeneities are most likely caused by small defects in the aligning layer that result in the pinning of defects in the director organization. Because of the large-scale nature of the inhomogeneities, we do not expect them to play a significant role in the early-time evolution of 
the system. However, they do prevent detailed studies of the late-time ordering at this point, and the influence of these domains can not be completely ruled out for the early time evolution.

For each of the quenches, we measure a series of 128 images taken $1 \mathrm{~s}$ apart. We characterized the images using the spatial Fourier transform of each image. We focus on the square of the modulus of the Fourier transform of each image (the power spectrum, $S(k, \theta)$, where $k$ is the wavenumber of interest). The power spectra of 20 jumps are averaged image by image to improve the statistics. There is a 15 minute wait between jumps. Using $S(k, \theta)$, we measure the total power $P=\int_{0}^{2 \pi} \int_{0}^{\infty} S(k, \theta) k d k d \theta=$ $2 \pi \int_{0}^{\infty} S(k) k d k$, where $S(k)$ is the azimuthal average of $S(k, \theta)$. From this measure, we confirmed that the total power is essentially constant throughout the time evolution, allowing comparison between features of the power spectra at different times. The average wavenumber,

$$
<k>=\frac{2 \pi \int_{0}^{\infty} S(k) k^{2} d k}{P}
$$

is used as a measure of the typical length scale in the system. The rate of growth of this length scale can be compared for the two quenches using the time-dependence of the average wavenumber. Because we are looking at early time dynamics, it is not necessarily expected that a scaling regime will exist. However, we tested for scaling of the power spectrum of the form $S(k, t)=L^{d} g(k L)$, where $L$ represents a typical length scale for the system and $d$ is the spatial dimension of the pattern. For our case, $d=2$, and we take $L=\langle k\rangle^{-1}$. Finally, in the study of phase ordering, Porod's Law 2, 21] states that in the limit of large wavelength $(k L>>1), S(k, t) \sim L^{-n} k^{-(d+n)}$. In this case, $d=2$ and $n$ is the dimension of the vector order parameters that describes the system. For a nematic liquid crystal, one expects $n=2$. In this sense, Porod's Law relates the behavior of the long wavelength tail of the power spectrum to the dominate defects in the system [2].

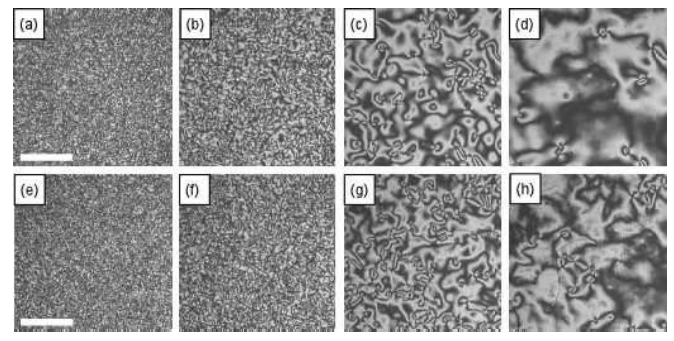

FIG. 2: Series of images for the frequency (a - d) and voltage $(\mathrm{e}-\mathrm{h})$ quenches. The white bar in the images represents $1 \mathrm{~mm}$. The images are taken at $1 \mathrm{~s}, 4 \mathrm{~s}, 32 \mathrm{~s}$, and $128 \mathrm{~s}$ after each quench.

Figure 2 presents the typical time evolution of the system after a quench in voltage. Images $2(\mathrm{a})-(\mathrm{d})$ are for a frequency quench, and images $2(\mathrm{e})-(\mathrm{h})$ are for a voltage quench. The images are taken at $1 \mathrm{~s}, 4 \mathrm{~s}, 32 \mathrm{~s}$, and $128 \mathrm{~s}$ after the quench and illustrate the ordering that occurs. The images suggest that the voltage quench evolves slower than the frequency quench. To quantify this difference, we used the time dependence of $\langle k\rangle$.

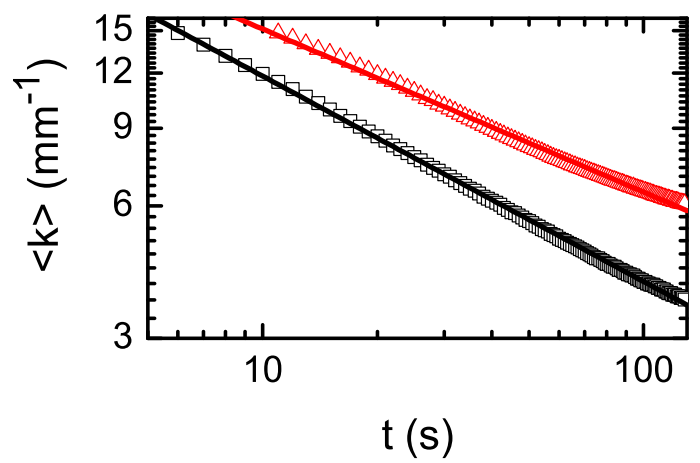

FIG. 3: Plot of the average wavenumber versus time for each of the quenches. The frequency quench is represented by squares and the voltage quench is represented by triangles. The solid line in each case is a fit to a power law. The exponent for the frequency quench is -0.47 and for the voltage quench, it is -0.37 .

The main result of this paper is shown in Fig. 3. Plotted in Fig. 3 is the average wavenumber, $k$, versus time for both quenches. The most dramatic feature of this result for the frequency quench is the agreement with power law growth over the entire time range. The voltage quench is also consistent with power law growth for the time range plotted in Fig. 3. By comparing the exponents in the power law (-0.47 for the frequency quench and -0.37 for the voltage quench), one finds that the system orders faster after a frequency quench than it does after a voltage quench.

The existence of power law behavior for the evolution of the average wavenumber suggests that the system might be in a scaling regime. The limited time of observation makes it difficult to establish this with any certainty. However, as a test of scaling, we considered the behavior of the full power spectra. The results for scaling the azimuthally averaged power spectra are shown in Fig. 4. The results are consistent with a scaling of the power spectra, but are not conclusive. Perhaps more important than the overall behavior of the spectra is the behavior at large wavenumber. This is shown in the insert of Fig. 5 for a time $16 \mathrm{~s}$ after the quench. The long wavelength part of the power spectra is consistent with a power law that is independent of the quench type: $S(k) \sim k^{-4}$ for large $k$. This result is in agreement with the expected value for ordering of a nematic liquid crystal in two dimensions [2].

To summarize, the two quenches started from different values of the average tilt of the director. Because the average wavenumber was consistent with a power law, the different rates of phase ordering could be quantified. 


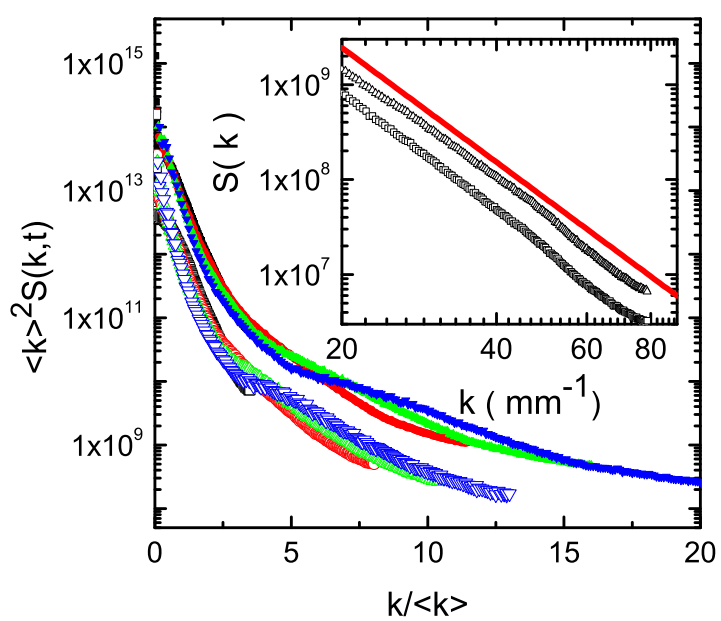

FIG. 4: Plot of the scaled power spectra versus the average wavenumber for the frequency quench (solid symbols) and for the voltage quench (open symbols). The power spectra for $2 \mathrm{~s}$, $32 \mathrm{~s}, 64 \mathrm{~s}$, and $128 \mathrm{~s}$ after the quench is shown for each case. The insert shows the long time tail for $16 \mathrm{~s}$ after the frequency quench (squares) and the voltage quench (triangles). The solid line represents $k^{-4}$ and is provided as a guide to the eye.

The frequency quench (with the same initial and final tilt) exhibited faster ordering than the voltage quench. This is consistent with the different intrinsic time scales for electroconvection. The two main relaxation times are the charge relaxation time (on the order of $10^{-3} \mathrm{~s}$ for this system) and the director relaxation time (on the order of $1 \mathrm{~s}$ for this system). Because the voltage quench should be dominated by director relaxation at early times as the system equilibrates to the equilibrium tilt angle, it is not too surprising that this quench has slower dynamics.

Of interest for future work is the behavior of the exponents that correspond to the power law growth of the average wavenumber. For this system, the order parameter is not conserved, so one would expect an exponent of $1 / 2$. This work was a focused study that compared frequency quenches and voltage quenches. For the frequency quench, the scaling of the average wavenumber was close to $1 / 2(0.47)$, but for the voltage quench, it was significantly smaller (0.37). It should be noted that for the voltage quench, by considering different time windows $(0.1 \mathrm{~s}, 0.5 \mathrm{~s}$, and $1 \mathrm{~s})$, we observed apparent exponents for the evolution of $\langle k\rangle$ of $0.28,0.35$, and 0.37 , respectively. For the frequency quenches, the exponents were essentially constant. This suggests two things. The time dependence of the evolution of the two systems may become similar in the long-time limit, if the trend for the voltage quench continues. If that turns out to be true, both systems obey the expected growth laws for an equilbrium system at late times. However, more uniform systems and longer times are needed to confirm this behavior, with a focus on the voltage quenches for which the behavior is the most dramatic. Additionally, the impact of the type of quench can be further explored using mixed quenches where both the frequency and voltage is varied.

\section{Acknowledgments}

This work was supported by NSF grant DMR-9975479 and PRF 39070-AC9.
[1] M. C. Cross and P. C. Hohenberg, Rev. Mod. Phys. 65, 851 (1993).

[2] A. J. Bray, Advances in Physics 43, 357 (1994).

[3] K. R. Elder, J. Vinals, and M. Grant, Phys. Rev. Lett. 68, 3024 (1992).

[4] M. C. Cross and D. I. Meiron, Phys. Rev. Lett. 75, 2152 (1995).

[5] J. J. Christensen and A. J. Bray, Phys. Rev. E 58, 5364 (1998).

[6] D. Boyer and J. Vinals, Phys. Rev. E 65, 046119 (2002).

[7] H. Qian and G. F. Mazenko, Phys. Rev. E 67, 036102 (2003).

[8] H. Qian and G. F. Mazenko, Phys. Rev. E 69, 011104 (2004).

[9] D. Boyer, Phys. Rev. E 69, 066111 (2004).

[10] L. Purvis and M. Dennin, Phys. Rev. Lett. 86, 5898 (2001).

[11] C. Kamaga, D. Funfschilling, and M. Dennin, Phys. Rev. E 69, 016308 (2004).

[12] C. Kamaga, F. Ibrahim, and M. Dennin, Phys. Rev. E 69, 066213 (2004).
[13] C. Harrison, Z. Cheng, S. Sethuraman, D. A. Huse, P. M. Chaikin, D. A. Vega, J. M. Sebastian, R. A. Register, and D. H. Adamson, Phys. Rev. E 66, 011706 (2002).

[14] L. Kramer and W. Pesch, Annu. Rev. Fluid Mech. 27, 515 (1995).

[15] P. G. de Gennes and J. Prost, The Physics of Liquid Crystals (Clarendon Press, Oxford, 1993).

[16] Y. Hidaka, J. H. huh, K. Jayaski, M. I. Tribelsky, and S. Kai, J. Phys. Soc. Japan 66, 3329 (1997).

[17] P. Toth, A. Buka, J. Peinke, and L. Kramer, Phys. Rev. E 58, 1983 (1998).

[18] E. Industries, Technical Documentation (2001).

[19] J. Collins, D. Funfschilling, and M. Dennin, Thin Solid Films p. submitted (2005).

[20] M. Dennin, Phys. Rev. E 62, 6780 (2000).

[21] H. K. Janssen, B. Schaub, and B. Schmittmann, Zeitschrift fur Physik B (Condensed Matter) 73, 539 (1989). 